\title{
COMBINING DISTRIBUTED AND CENTRALISED APPROACHES TO DEVELOP A HYBRID METHODOLOGY FOR DATA MANAGEMENT IN HEALTHCARE
}

\author{
Christos Kontzinos ${ }^{1}$, Panagiotis Kapsalis, Spiros Mouzakitis, Michael Kontoulis, Ourania Markaki, \\ Dimitris Askounis, Haralampos Karanikas ${ }^{2,3}$, Alexandros Christodoulakis ${ }^{3}$ \\ and Panagiotis Dimitrakopoulos ${ }^{3}$ \\ ${ }^{1}$ Decision Support Systems Lab, National Technical University of Athens, Athens, Greece \\ ${ }^{2}$ Department of Computer Science and Biomedical Informatics, University of Thessaly, Athens, Greece \\ ${ }^{3}$ Datamed SA, Athens, Greece
}

\begin{abstract}
In the $21^{\text {st }}$ century, the combination of rapid technological developments as well as the adoption of emerging technologies has impacted almost every area of everyday life. These developments have even affected domains that traditionally operate without the help of Information and Communication technologies (ICT) but now see an increase in digitization of their processes. Healthcare is one of those domains that is being revolutionized via the emergence of the e-health and m-health paradigms as well as the automation of medical processes, robotics, and Internet of Things (IoT) medical devices and sensors. These developments, apart from modernizing healthcare and providing quality-of-life solutions to both healthcare practitioners and patients, have also led to an increase in the availability of digital medical data, which are nowadays being structured in Electronic Health Records (EHRs). EHRs contain various health data about a patient and are the basis for the development and implementation of various innovative solutions in healthcare. Such solutions mainly aim to address some of the current shortcomings of EHRs such as the centralized storage, which makes them vulnerable to mass hacker attacks as well as the lack of interoperability among various medical standards that are used to structure EHRs, which makes it hard to manage and share them across different healthcare providers. The scope of this publication is to introduce blockchain technology as a potential solution to the aforementioned challenges and present a novel methodology and architecture that have been developed to be implemented in a healthcare application. The methodology presented in this paper leverages both blockchain and centralized databases, making it a hybrid approach but aims to set the basis for developing blockchain-powered healthcare solutions for data management. Under that context and the need to combine blockchain with centralized databases, the security parameters of the system are also tailored to work specifically with that kind of approach. The end-goal of the methodology and architecture presented in this publication is to give patients ownership of their data, help them manage and share them as they see fit, while at the same time keeping in mind that large healthcare providers must have access to the same information in a secure manner for the well-being of their patients. It is considered that this hybrid approach offers a well-tailored solution for both stakeholders.
\end{abstract}

\section{KEYWORDS}

Blockchain, Healthcare, Electronic Health Record, Key Management, Consensus, Hash

\section{INTRODUCTION}

In the fast evolving $21^{\text {st }}$ century, the rapid development of innovative Information and Communication Technologies (ICT) has revolutionized almost every aspect and task of everyday life. In addition, automation and the introduction of the Internet of Things have introduced and generated in a similar way a wealth of digital data. The aforementioned, combined with the rapid increase in database capacity as well as innovative storage and processing technologies, create opportunities for digitization in various sectors through the development of ICT-powered applications. Especially in health, automation of medical examinations, robotics and self-diagnosis have already led to improved population care, better patient experience and the production of large amounts of medical data. Medical or health data are defined as any data relating to health conditions, the effects of the reproductive process, the causes of death and the general quality of life for an 
individual or a population group (Segen 2006). Such data are collected when individuals interact with healthcare applications or hospital administration systems and usually include a record of the services offered, the conditions under which they were provided and the clinical outcomes or other information related to those medical services (Tzourakis 1996). Medical data are nowadays increasingly organized and structured through Electronic Health Records (EHRs), which emerged as a direct result of the aforementioned developments and aim to leverage the opportunities afforded by the increasing digitization in the health sector. EHRs are the systematic collection of patient health information in electronic form (Gunter and Terry 2005) and include data such as demographics, medical history, allergies and medication, laboratory test results, vital signs and personal statistics among others (Top Mobile Trends 2014). EHRs can in theory be shared among digital applications of different healthcare organizations (hospitals, insurance companies, etc.) and individuals (doctors, patients, etc.) and are considered to be a critical factor in improving the intelligence and quality of medical procedures (Yue et al. 2016), as well as reducing healthcare costs (Kemkarl and Dahikar 2012). In fact, the exchange of medical data between different EHR systems can provide a better understanding of public health standards and trends (Ge et al. 2013), which can in turn offer better quality of care (Jothi et al. 2015), personalized referrals for patients (Zhang et al. 2017) and increased efficiency in health care provision both at a national and global level. The structuring of medical data for EHR systems is achieved through medical standards, some of the most popular ones being Health Level 7 or HL7 (HL7 International 1989), Fast Healthcare Interoperability Resources or FHIR (HL7 International 2018), and openEHR (openEHR Foundation 2018).

Currently, the biggest challenge for the widespread adoption of EHR systems is finding ways to collect, store and analyze sensitive health data without raising concerns about the patients' privacy (Clifton et al. 2013). According to a report produced by the ONA General Council (ONA General Council 2016), the lack of adequate measures to safeguard patient privacy has led to many breaches of EHR systems, which leave patients exposed to profiling and exploitation by malicious users. Such violations, in addition to impacting patient care, usually have financial and legal consequences for health care providers as well (Peterson et al. 2016). In fact, securing EHRs, while also keeping them shareable among different applications is hard to achieve with traditional approaches and the consequences of breaches in such systems are a strong disincentive to the exchange of data between different providers (Barrows and Clayton 1996). Another major obstacle to the exchange of medical data is the lack of agreement regarding technical requirements and specifications on the infrastructure that will be used. In addition, most healthcare systems require either a centralized data source or bulk data transmission from one system to another. However, centralized data storages increases the risk of massive eavesdropping and malicious attacks, while bulk sending medical data requires organizations to authorize the use and processing of data to other healthcare providers, which may breach their terms of services with the patients depending on the data usage and processing activities by the third party.

In conclusion, although EHRs offer many possibilities, due to the aforementioned challenges, they have not been widely applied in the healthcare domain. One technology that can provide solutions and reduce or even eliminate the disadvantages of existing systems and applications is blockchain. Blockchain is based on distributed data storage, which can reduce the cost of implementing new systems, while the security it offers is greater than that provided by existing solutions. In this context, the purpose of this study is to explore existing applications of blockchain in healthcare and most importantly present a hybrid methodology that combines blockchain and traditional databases for the effective and secure management, sharing and processing of sensitive medical data. This methodology is designed as part of the Impilo research project, which aims to develop an innovative solution for the healthcare domain, building on blockchain and social media practices. The platform will consist of a blockchain ledger, which will act as a healthcare data repository for the construction and retrieval of EHRs, as well as the Impilo application, which will offer added-value services on top of the blockchain ledger and manage the interactions and data transactions among doctors and patients.

Section 1 introduces the scope of this paper by presenting the current situation and challenges arising from the lack of suitable technical solutions in the healthcare domain. Section 2 includes a short state-of-the-art analysis regarding current blockchain approaches in healthcare. Section 3 presents in detail, Impilo's methodology for healthcare data management that is based on blockchain. Finally, Section 4 concludes the document and details steps for future work. 


\section{RELATED WORK}

The main scope of this section is to present blockchain-based research approaches that have been designed and developed to provide efficient and secure exchange of medical information between patients, physicians and other organizations. Peterson et al. (2016) designed a hybrid approach to developing a healthcare blockchain that makes use of the rising FHIR standard for structuring medical information. In this system, the network nodes represent patient data transactions and describe the addition of a resource (i.e., a specific medical information) to the official patient file. Transactions, however, do not include the actual medical information record document. Instead, they point to where the FHIR resources are stored via URL pointers, and that is where the system hybridizes between the blockchain and the central databases. The way the system is structured allows healthcare providers to maintain functional control of their data as it maintains sensitive patient data outside of the blockchain. Yue et al. (2016) developed a blockchain-based architecture that allows the patients to own, control, and share their medical data with ease and security. The system collects the patients' medical data from a variety of systems (medical records information systems, data analysis algorithms, etc.) and allows them to manage them in a unified manner via a personalized data portal. The data is stored in a private blockchain cloud, which ensures, through various cryptographic techniques that they cannot be accessed or altered by anyone, including physicians and patients, that have no authorization on a given patient's medical record. MedRec (Ekblaw et al. 2016) is a decentralized blockchain-based electronic health record system that provides patients with a complete and immutable medical diary that allows them to have easy access to their medical information regardless of their healthcare provider(s). By utilizing the inherent features of blockchain, MedRec offers accessibility, confidentiality and secure data exchange, which are critical issues when managing sensitive medical data. Users of the system can view and validate themselves the authenticity and history of their data and manage access rights to it, while the system has a variety of APIs that allow it to integrate with existing databases of various health care providers. In general, it can be observed in the respective research literature that the general case of blockchain use in healthcare is within the framework of the techniques already presented. In fact, in a relevant publication by Gordon and Catalini (2018), all the ways and methodologies for patient-driven healthcare interoperability were studied and presented, based on existing and emerging technologies, aiming to improve the management of medical information in healthcare. For blockchain-based systems, the general case is the one that has been presented in the current section. It is therefore concluded that in the field of health the use of blockchain is mainly intended to add a layer of security to patients' sensitive data, allow them to manage access rights on them, and facilitate communication between different organizations that provide medical services to patients. In the general case, the actual patient data is stored either in centralized databases of various healthcare organization or in distributed data wallets. However, the blockchain in these systems helps patients verify the authenticity of their information and view their medical history through a trusted digital trail.

\section{BLOCKCHAIN-BASED METHODOLOGY FOR DATA MANAGEMENT IN HEALTHCARE}

This section presents Impilo's blockchain-based methodology for data management in healthcare and describes not only structural (block structure, system architecture) but also security parameters (hash function, key management, consensus) of the projected system. The main differentiation between Impilo and the approaches presented in the previous section lie in the fact that Impilo will develop the blockchain ledger based on the new Hyperledger Iroha platform (Hyperledger 2019) that is specifically tailored for development of non-cryptocurrency data management solutions and has built-in algorithms that deal with various shortcomings and bottlenecks of other platforms.

\subsection{Hybrid Methodology for Data Management}

\subsubsection{Block Structure}

This section, taking into account the respective literature and the requirements of the projected Impilo platform, presents the structure of any given block of the Impilo blockchain as well as the information that 
will be contained within (see Figure 1). In general, the structure of the Impilo blockchain is based on Merkle trees. In computer science, a Merkle tree or hash tree is a tree that labels leaf nodes with the cryptographic hash of a block of data and non-leaf nodes with the cryptographic hash of its child nodes. Merkle trees allow for the efficient and secure verification of the contents of large data structures (Becker 2008). In the Impilo system, the nodes of the Merkle tree represent patient data transactions and describe the addition of a resource (i.e., specific medical information) to the official patient record. The hashes of all transactions in a block contribute to the hash of the Merkle root, or block header, which contains the following metadata used to validate each new block: the hash of the block, the hash of the previous block (for validation purposes), the subscriber signatures of each node that contributed to the block (to ensure that the block will remain valid after being assembled by the respective miner) and the miner elections (in which each node that contributed to the block is required to provide a random number encrypted with the node's private key to prepare for the next miner election).

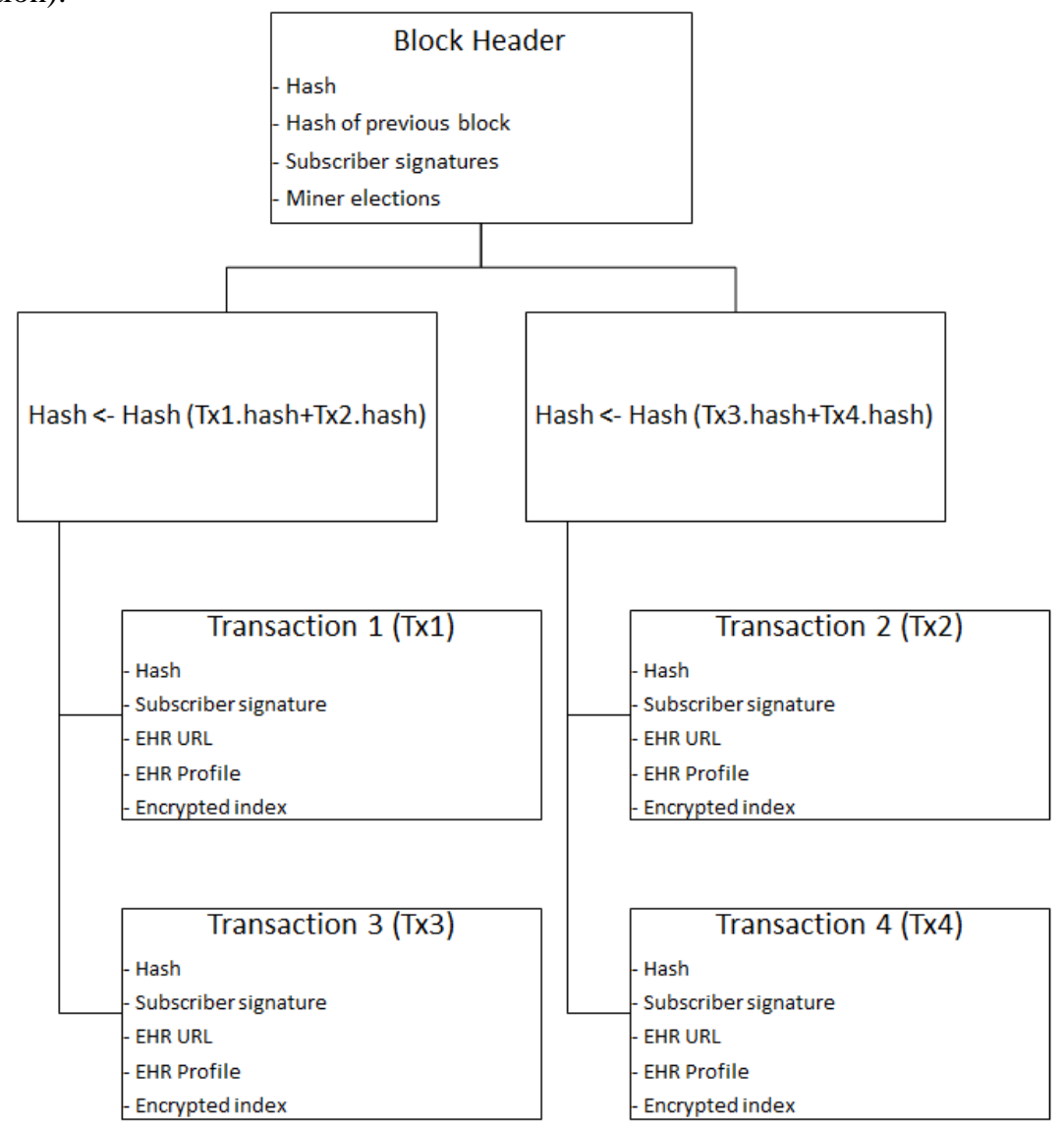

Figure 1. Block structure

In this approach, a medical transaction stored in a blockchain includes the following information (see Figure 1): the transaction hash (although the resource is not inserted into the blockchain, its contents can be verified using the transaction hash during retrieval), the signature of origin (digital signature of the source node), the URL of the EHR (link to the actual location of the EHR resource where the data is located), the profile of the EHR (URI of the EHR profile to which this resource corresponds) and, finally, an encrypted index that allows data to be discovered without leaking file information. Although the block itself does not contain the actual data, it must provide search and tracking capabilities, as well as a data access mechanism once the appropriate transactions have been found. External entities, with the appropriate permissions, can query the blockchain, using keywords in the encrypted index field of the transaction. These keywords can be searched despite the fact that they are encrypted to avoid data leakage (Ioannidis 2005). 


\subsubsection{System Architecture}

In Impilo patient data will be stored in an external database, as previously mentioned, while blockchain will be used to validate and store transactions. This sub-section presents the high-level architecture of the projected system (see Figure 2). The Impilo high-end architecture consists of three individual elements. Firstly, the client is the Impilo application through which users will have access to the various functions that will be developed, the blockchain database and the central database. Following that, the blockchain repository will be used to validate and store transactions related to a patient's health data. A sufficient number of computer nodes will be employed for storing the Impilo blockchain in a distributed manner so that users' personal (portable and non-portable) devices are not burdened with storing the entire chain, but at the same time maintain access to it. This also increases the likelihood of some malicious action being detected. Finally, the central database is where the actual medical information will be stored. To enhance system security, the data will be encrypted to preserve their integrity and prevent their processing by third-party malicious users.

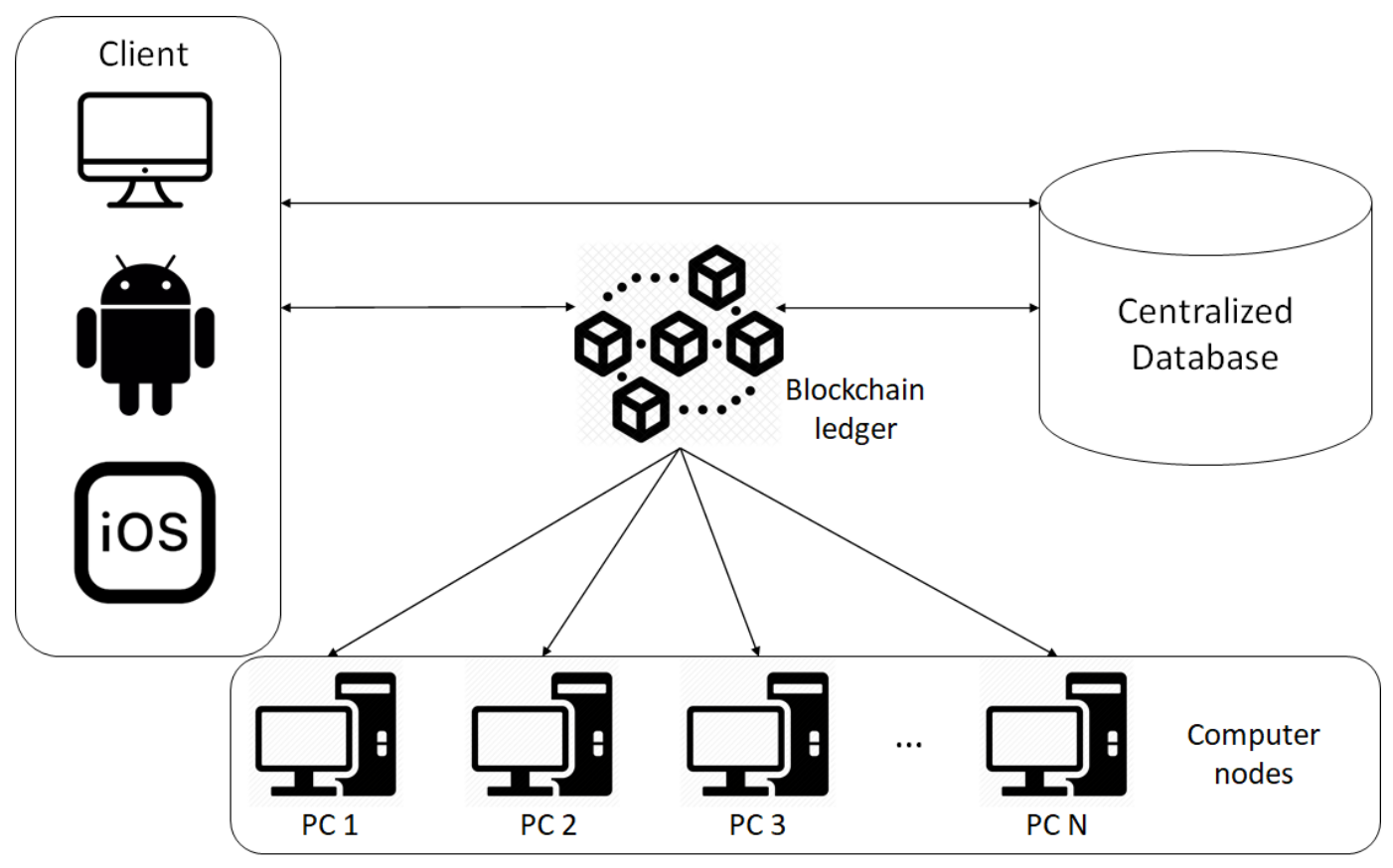

Figure 2. System architecture

The operation of the system can be made clear through the following example. Suppose an Impilo user wants to save his blood tests in his medical file. He logs in to the Impilo application with his credentials and performs the actions required to store new information in his medical file. The Impilo client communicates with the central database and stores the information in the patient's file. The blockchain ledger communicates with both the client and the database to generate the hash that will be needed to store the transaction details in a block. After storing the new information, the client and the database communicate with the blockchain, verify that the hash is the same in both of them and if it is, the transaction details are stored in a blockchain block. It is worth noting that to increase overall security, patient data in the central database will be hashed and the decryption key will be the users' password with which they access the Impilo application. This means that only users can decrypt their data when accessing the application and in case of a malicious attack on the system, the attacking party will have to know the passwords of the patients in order to receive the correct information.

\subsection{Hash Function, Key Management and Consensus Approach}

In general, blockchain systems use encryption techniques to ensure the integrity of their content and to detect breaches. For increased data protection, Merkle trees are used to build blocks. In this case, any change in the system state leads to a new hash value of the root node. This way, all nodes in the system are notified of the change in a block and can approve or reject the change. If a system state change is accepted by its nodes, it is 
attached to the network in the form of a new immutable block (Mohanta et al. 2019). In the following sub-sections, Impilo's following security parameters will be presented: selected hash function, key management system and consensus algorithm. It should be noted that the Impilo application will be built on the Hyperledger Iroha platform that works with specific options for hash functions, key management systems and consensus algorithms. As such and given the platform requirements, an assessment of various blockchain platforms was performed to identify the one that best suits the needs of the projected application. Hyperledger Iroha was selected because it is mainly used for digital data management, has a robust permission system, gives users the ability to manage access rights to their data based on predefined commands and is the easiest one to set up in a real-life scenario. As such, the security parameters described in the following sub-section follow the general guidelines of Hyperledger Iroha, tailored though for a healthcare blockchain system.

\subsubsection{Hash Chains}

A hash chain is the sequential application of a cryptographic hash function to a piece of data. In computer security, a hash chain is a method for generating multiple keys from a single key or password. These keys can be used once each to gain access to the data concerned. A hash function can also be applied sequentially to additional pieces of data to keep records of the data history. Cryptographic hash functions allow the hash to be calculated easily. However, it is considered difficult - practically impossible - to calculate the data from which it emerged. Hash chains are similar to blockchains, as in both cases cryptographic hash functions are used to connect two nodes. However, a blockchain generally aims to support distributed consensus across a network and incorporates a set of rules for encapsulating data and related permissions for accessing and managing them. In blockchain, hash algorithms are used to determine the unique state of the system at any given time. The blocks are linked data ledgers, while, as mentioned above, the block header includes a hash pointing to the previous block of the chain. The blocks are connected to each other via hashes, which represent data within the previous blocks along with their addresses. The best-known cryptographic functions are MD5, SHA-1, SHA-2 and SHA-3 that are widely used in various projects. However, under the scope of the Impilo project, the BLAKE2 hash function is employed. BLAKE2 is a cryptographic hash function that is faster than MD5, SHA-1, SHA-2 and SHA-3, and as secure as the latest SHA-3 standard. BLAKE2 has been adopted by many projects due to its high speed, safety and simplicity.

\subsubsection{Key Management}

Blockchain employs public key cryptography or asymmetric cryptography, which was invented in the late 1970s (Diffie and Hellman 1976) and provides a completely different model of encryption key management from earlier symmetric key encryption methods. The basic idea is that the sender and recipient do not share a common secret key as in the case of symmetric key encryption but have different keys for different functions. Specifically, each user has two encryption keys: one is called a private key and the other a public key. These two keys (private and public) are mathematically related to each other. If one is used to encrypt a message, then the other is used to decrypt it. The success of this type of cryptographic algorithm is based on the fact that knowledge of the public encryption key does not in any way allow the calculation of the private encryption key. The public key can be disclosed to third parties, and is used to encrypt data, while the private key is used to decrypt it (Stallings 1998). The blockchain security model assumes the existence of public key encryption. Identities, including user identities and transactions, are derived from public key certificates. Therefore, secure key management is essential for every blockchain project. As already mentioned, the Impilo project will specifically use Hyperledger Iroha, which is a platform for developing blockchain applications. Therefore, the management of public and private keys will follow the instructions of this platform's documentation.

\subsubsection{Consensus Algorithm}

The role of consensus in blockchain systems is to ensure that all non-faulty network users perform the same system status updates in the order in which the events that changed the status occurred. Achieving consensus on decentralized systems, such as the Impilo blockchain, which receive asynchronous events and are subject to "Byzantine Fault Tolerance" is a common challenge in many applications. The YAC (Yet Another Consensus) algorithm (Muratov et al. 2018) will be used to reach consensus on the Impilo system. YAC is a practical decentralized consensus algorithm, which attempts to solve traditional problems in Byzantine Fault Tolerance, such as inefficient message transmission and the fact that the system is dependent on "powerful leaders". Powerful leaders in a blockchain system (i.e., users who occupy many of its nodes) make sense in a cryptocurrency blockchain. However, a medical blockchain must ensure independence of users from the 
"leaders" of the system. YAC is actually used to provide Byzantine consent to the Hyperledger Iroha Blockchain project and empirical results show that this solution achieves low system inertia, which leads to high transaction efficiency.

YAC operates in the following way. Initially, typical YAC participants include the client, peer and ordering service. Every client is essentially a user who has a public key registered in the blockchain. The client's role lies in generating transactions and sending them to the ordering service. The client is also able to develop smart contracts that are used to define the rights of other users on his/her data as well as to query the other peers. A peer is one of the network participants who are responsible for validating and reaching an agreement regarding the transactions that have transpired and their storage in the system's blocks. Peers maintain a complete digital trail of transactions in the system and validate new block proposals. Finally, the ordering service is a unit that receives a set of transactions and generates new block proposals. A block proposal contains a list of transactions, which must be accepted by the peers.

For the application of YAC in the Impilo system, the following set of rules will be followed to better explain the consensus algorithm. Firstly, the client will be known to the peers and each client can interact with a specific list of peers. In addition, Impilo users will be incentivized to store their keys on a device, such as their mobile phone. Finally, the client will have the rights to execute a specific subset of orders/smart contracts, such as creation of a transaction. The high-level operating system flow can be described by the following steps. Initially, a client creates a transaction through orders that he/she has the right to execute and signs it with his/her private key. The client then sends the transaction to a peer, who receives it, validates it to ensure it is in the correct form (as this is defined by the system) and transfers it to the ordering service. Finally, the ordering service forms a block proposal, which contains a list of transactions that will potentially be added to the blockchain. The block proposal can only be formed if the ordering service has collected a certain number of transactions or after a specific time period and is then sent to all system peers. Following that, the peers exchange votes through the system and finally decide on the new block. In essence, at this stage, each peer must calculate whether the block proposal (set of transactions) sent by the ordering service is valid. The block is created by the peer and contains a suggested hash, the transactions of the block proposal and the data required for the cryptographic validation of the blockchain. The suggested hash defines a unique proposition for each transaction round as it represents the peer's intention to store a set of transactions in the chain. These hashes are an integral part, as it is possible that different peers calculate different blocks from a proposal. Next, peers must send in their vote via a message that contains the aforementioned hashes as well as a signature that verifies the peer. The order in which peers will vote for a block hash is determined via a permutation function. During the voting process, the message is sent to the first peer of the list and then to the next one either after the first sends a vote, or after a certain period of time. The process continues until the network receives a valid message accepting or rejecting the changes. Network consensus requires at least $2 / 3$ of the network. When a peer receives the majority of the network it transmits an acceptance message to the others. Finally, the peer to whom the transaction was originally sent saves the block in its local copy.

\section{CONCLUSION}

The purpose of this publication was the analysis of the research field regarding the use of blockchain in healthcare, as well as the presentation of a novel methodology and architecture that have been developed to facilitate data management in the domain. Initially, the relevant literature studied supports the application of blockchain technology in conjunction with popular medical standards and traditional approaches for structuring medical information. In the technical part of the publication, research approaches on the construction of medical data via blockchain were presented and then the methodology and architecture that Impilo will follow for the construction of medical information were described. The network on the one hand benefits from the blockchain properties for security and stability and on the other hand is flexible in the requirements of the users by leveraging central databases that can facilitate faster data transactions. The last section of the publication describes the techniques that will be used to secure health data and, more specifically, the hash functions, key management and network consensus. Overall, it can be said that the application of blockchain technology in areas other than cryptocurrencies is of particular value. This is evident from the ever-increasing research interest in blockchain, in areas where it has traditionally not been implemented. Especially in the health sector, where Impilo operates, blockchain may create more reliable and resilient networks, while facilitating seamless communication between the various stakeholders (doctors, patients, hospitals, etc.). The next steps of the Impilo research project include the development of the system and its pilot application to specific healthcare providers and a sample of patients. 


\section{ACKNOWLEDGEMENT}

The IMPILO project is funded by the Greek operational program "Competitiveness, Entrepreneurship, Innovation" - EPANEK (2014-2020), in the national action: "RESEARCH - CREATE - INNOVATE", with project code T1EDK-01382.

\section{REFERENCES}

Barrows R.C. Jr and Clayton P.D., 1996, Privacy, confidentiality, and electronic medical records. Journal of the American Medical Informatics Association. No 3, pp 139-148.

Becker G., 2008, Merkle signature schemes, merkle trees and their cryptanalysis. Ruhr-University Bochum, Tech. Rep.

Clifton C. et al, 2004, Privacy-preserving data integration and sharing. Proceedings of the 9th ACM SIGMOD Workshop on Research Issues in Data Mining and Knowledge Discovery. Paris, pp 19-26.

Diffie W. and Hellman M.E., 1976, Multiuser cryptographic techniques. Proceedings of the June 7-10, 1976, national computer conference and exposition.

Ekblaw A. et al, A case study for blockchain in healthcare: "MedRec" prototype for electronic health records and medical research data. Proceedings of IEEE Open \& Big Data Conference. Washington, DC, pp 13.

Ge Y. et al, 2013, Patient-controlled sharing of medical imaging data across unaffiliated healthcare organizations. Journal of the American Medical Informatics Association. No 20, pp 157-163.

Gordon W.J. and Catalini C., 2018, Blockchain technology for healthcare: Facilitating the transition to patient-driven interoperability. Computational and structural biotechnology journal. No 16, pp 224-230.

Gunter T.D. and Terry N.P., 2005, The emergence of national electronic health record architectures in the United States and Australia: Models, costs, and questions. Journal of medical Internet research. No 7(1), e3.

HL7 International, 1989, Health level 7. 1st ed. HL7. Available at: http://www.hl7.org/implement/standards/.

HL7 International, 2018, Fast Health Interoperability Resources (FHIR). 4th ed. HL7. Available at: https://www.hl7.org/fhir/.

Hyperledger, 2019, Hyperledger Iroha documentation. Available at: https://iroha.readthedocs.io/en/latest/.

Ioannidis J., 2005, Applied cryptography and network security. ACNS, New York, NY.

Jothi N. et al, Data mining in healthcare - A review. Procedia computer science. No 72, pp 306-313.

Kemkarl O.S. and Dahikar D.P.B., Can electronic medical record systems transform health care? Potential health benefits, savings, and cost using latest advancements in ict for better interactive healthcare learning. International Journal of Computer Science \& Communication Networks. No 2, pp 453-455.

Mohanta B.K. et al, 2019, Blockchain technology: A survey on applications and security privacy challenges. Internet of Things. No 8, pp 100-107.

Muratov F. et al, 2018, BFT consensus algorithm for blockchain. arXiv preprint arXiv:1809.00554.

Ontario Nurses' Association General Council, 2016, Members and patient privacy: Be aware and beware! ONA, Toronto. Available at: https://www.ona.org/.

openEHR Foundation, 2018, OpenEHR. 3rd ed. Available at: https://www.openehr.org/

Peterson K. et al, 2016, A blockchain based approach to health information exchange networks. Proceedings of NIST Workshop Blockchain Healthcare. Gaithersburg, pp 1-10.

Segen J.C., 2006, Concise dictionary of modern medicine. McGraw-Hill Education, New York.

Stallings W., 1998, Cryptography and network security: Principles and practice. 2nd ed. Prentice-Hall Inc, Upper Saddle River, NJ.

Top Mobile Trends, 2014, Mobile tech contributions to healthcare and patient experiences. Available at: http://topmobiletrends.com/ mobile-technology-contributions-patient-experience-parmar/.

Tzourakis M.C., 1996, The healthcare industry and data quality. Proceedings of the 1996 International Conference on Information Quality. pp 87-93.

Yue X. et al, 2016. Healthcare data gateways: Found healthcare intelligence on blockchain with novel privacy risk control. Journal of medical systems. No 40, pp 218.

Zhang Y. et al, 2017, iDoctor: Personalized and professionalized medical recommendations based on hybrid matrix factorization. Future Generation Computer Systems. No 66, pp 30-35. 